\title{
Analytic second-order energy derivatives in natural orbital functional theory
}

\author{
Ion Mitxelena ${ }^{1}$ and Mario Piris ${ }^{1,2}$ \\ ${ }^{1}$ Kimika Fakultatea, Euskal Herriko Unibertsitatea (UPV/EHU), 20080 Donostia, Euskadi, \\ Spain, and Donostia International Physics Center (DIPC), 20018 Donostia, Euskadi, Spain. \\ ${ }^{2}$ IKERBASQUE, Basque Foundation for Science, 48013 Bilbao, Euskadi, Spain.
}

\begin{abstract}
The analytic energy gradients in the atomic orbital representation have recently been published (J. Chem. Phys. 146, 014102, 2017) within the framework of the natural orbital functional theory (NOFT). We provide here an alternative expression for them in terms of natural orbitals, and use it to derive the analytic second-order energy derivatives with respect to nuclear displacements in the NOFT. The computational burden is shifted to the calculation of perturbed natural orbitals and occupancies, since a set of linear coupled-perturbed equations obtained from the variational Euler equations must be solved to attain the analytic Hessian at the perturbed geometry. The linear response of both natural orbitals and occupation numbers to nuclear geometry displacements need only specify the reconstruction of the second-order reduced density matrix in terms of occupation numbers.
\end{abstract}

\section{INTRODUCTION}

The matrix of second-order energy derivatives with respect to nuclear displacements, or just the Hessian, is directly related to many properties of great interest to chemists [1 5]. Derivative methods are widely used to characterize the stationary points on the potential energy surface, but are also essential for the study of highresolution molecular spectroscopy [] ], or geometry dependent molecular properties such as electrostatic moments [7]. Analytic first-order derivatives for reduced density matrix (RDM) methods are well-established, e.g. for the parametric second-order RDM method [22], as well as analytical expressions of second-order energy derivatives are well-known for standard electronic structure methods. Nevertheless, the latter are still missing for methods that have been appeared in the last few decades, such as those derived directly from RDMs [8 10, 19 21] without using the wavefunction.

In fact, the Hamiltonian corresponding to Coulombic systems only involves one- and two-particle operators, hence the ground-state energy of an electronic system can be computed using the first- and second-order RDMs, denoted hereafter as $\Gamma$ and $D$, respectively. Within the Born-Oppenheimer approximation, the electronic energy is then written as

$$
E_{e l}=\sum_{i k} \Gamma_{k i} \mathcal{H}_{k i}+\sum_{i j k l} D_{k l, i j}\langle i j \mid k l\rangle,
$$

where $\mathcal{H}_{k i}$ are the one-electron matrix elements of the core-Hamiltonian, whereas $\langle i j \mid k l\rangle$ are the two-electron integrals of the Coulomb interaction.

Accordingly, the role of the $\mathrm{N}$-particle wavefunction can be assumed by RDMs. Of particular interest are one-particle theories, where the ground-state energy is represented in terms of $\Gamma$, because the necessary and sufficient conditions that guarantee the ensemble Nrepresentability of $\Gamma$ are well established and are very easy to implement 11]. In addition, the unknown functional in a $\Gamma$-based theory only needs to reconstruct the electron-electron potential energy [12], which is a notable advantage over the density functional theory, where the kinetic energy functional needs also to be reconstructed. $\Gamma$-functional theories seem a promising way of overcoming the drawbacks of density functional approximations currently in use.

Most functionals employ the exact energy expression (1) but using solely a reconstruction functional $D[\Gamma]$. This implies that the exact ground-state energy will not, in general, be entirely rebuilt. Approximating the energy functional has important consequences [13. First, the theorems obtained for the exact functional $E[\Gamma]$ are no longer valid. The point is that an approximate functional still depends on $D$. An undesired implication of the $D$-dependence is that the functional N-representability problem arises, that is, we have to comply the requirement that $D$ reconstructed in terms of $\Gamma$ must satisfy the same N-representability conditions [, 19] as those imposed on unreconstructed second-order RDMs to ensure a physical value of the approximate ground-state energy. Otherwise, the functional approximation will not be correct since there will not be an $\mathrm{N}$-electron system with an energy value (1). In addition, due to this $D$-dependence, the resulting functional depends only implicitly on $\Gamma$ and is not invariant with respect to a unitary transformation of the orbitals.

Nowadays, the approximate functionals are constructed in the basis where $\Gamma$ is diagonal, which is the definition of a natural orbital functional (NOF). Accordingly, it is more appropriate to speak of a NOF rather than a functional of $\Gamma$ due to the existing dependence on $D$. In this vein, in the NOF theory (NOFT) [12], the natural orbitals (NOs) are the orbitals that diagonalize $\Gamma$ corresponding to an approximate energy expression, such as those obtained from an approximate wavefunction. The electronic energy can therefore be considered as a functional of the NOs and occupation numbers (ONs). In the 
following, we refer only to this basis, hence the groundstate functional for $\mathrm{N}$-electron systems is given by the formula

$$
E_{e l}=\sum_{i} n_{i} \mathcal{H}_{i i}+\sum_{i j k l} D\left[n_{i}, n_{j}, n_{k}, n_{l}\right]\langle i j \mid k l\rangle .
$$

In Eq. (2), $D\left[n_{i}, n_{j}, n_{k}, n_{l}\right]$ represents the reconstructed two-particle RDM in terms of the ONs. It is worth to note that we neglect any explicit dependence of $D$ on the NOs themselves because the energy functional has already a strong dependence on the NOs via the twoelectron integrals.

In the last two decades, much effort has been put into making NOFT able to compete with well-established electronic structure methods [10, 14]. In this vein, the analytic energy gradients in the atomic orbital representation for NOFT were obtained recently [15]. In the present paper, an alternative expression for them in terms of the NOs is given. On the other hand, the analytical calculation of second-order derivatives is also desirable over numerical treatment when high accuracy is required. Here, for the first time in the context of NOFT, the secondorder analytic energy derivatives with respect to nuclear displacements are given.

\section{THE HESSIAN}

The procedure for the minimization of the energy (2) requires optimizing with respect to the ONs and the NOs, separately. The method of Lagrange multipliers is used to ensure the orthonormality requirement for the NOs, and the ensemble N-representability restrictions on $\Gamma$, which reduce to $0 \leq n_{i} \leq 1$ and $\sum_{i} n_{i}=N$ [11]. The bounds on $\left\{n_{i}\right\}$ are enforced by means of auxiliary variables, so merely one Lagrange multiplier $\mu$ is needed to assure normalization of ONs. Hence, the auxiliary functional $\Lambda\left[\mathrm{N},\left\{n_{i}\right\},\left\{\phi_{i}\right\}\right]$ is given by

$$
\Lambda=E_{e l}-\mu\left(\sum_{i} n_{i}-N\right)-\sum_{k i} \lambda_{i k}\left(\left\langle\phi_{k} \mid \phi_{i}\right\rangle-\delta_{k i}\right) .
$$

By making (3) stationary with respect to the NOs and ONs, we obtain the Euler equations:

$$
\begin{gathered}
\frac{\partial E_{e l}}{\partial n_{m}}=\mathcal{H}_{m m}+\sum_{i j k l} \frac{\partial D_{k l, i j}}{\partial n_{m}}\langle i j \mid k l\rangle=\mu, \\
\frac{\partial E_{e l}}{\partial \phi_{m}^{*}}=n_{m} \hat{\mathcal{H}} \phi_{m}+\sum_{i j k l} D_{k l, i j} \frac{\partial\langle i j \mid k l\rangle}{\partial \phi_{m}^{*}}=\sum_{k} \lambda_{k m} \phi_{k} .
\end{gathered}
$$

Eq. (4) is obtained holding the orbitals fixed, whereas the set of the orbital Euler Eqs. (5) is satisfied for a fixed set of occupancies. For the sake of simplicity, we concern only on the use of real orbitals throughout this work. At present, the procedure of solving simultaneously Eqs. (44) and (5) is carried out by the iterative diagonalization method described in Ref. [16], which is based on the hermiticity of the matrix of Lagrange multipliers $\lambda$ at the extremum, i.e. $\left[\lambda-\lambda^{\dagger}, \Gamma\right]=0$ (where super-index $\dagger$ is used to express the conjugate transpose).

As it is shown in Ref. [15], the first-order derivative of the electronic energy with respect to Cartesian coordinate $x$ of nucleus $A$, written in the atomic orbital representation, reads as

$$
\begin{aligned}
\frac{d E_{e l}}{d x_{A}} & =\sum_{\mu v} \Gamma_{\mu v} \frac{\partial \mathcal{H}_{\mu v}}{\partial x_{A}}+\sum_{\mu v \eta \delta} D_{\eta \delta, \mu v} \frac{\partial\langle\mu v \mid \eta \delta\rangle}{\partial x_{A}} \\
& -\sum_{\mu v} \lambda_{\mu v} \frac{\partial \mathcal{S}_{\mu v}}{\partial x_{A}}
\end{aligned}
$$

so the energy gradient depends only on the explicit derivatives of one- and two-electron integrals and the overlap matrix. Therefore, there is no contribution from ONs, and the resulting Eq. (6) does not require obtaining the NOs and ONs at the perturbed geometry. One could differentiate Eq. (6) to achieve an expression for the Hessian, nevertheless, perturbation of both NOs and ONs must be considered. For that purpose it is more convenient to work in the natural orbital (NO) representation $\left\{\phi_{i}\right\}$, so that Eq. (6) transforms into

$$
\begin{aligned}
\frac{d E_{e l}}{d x_{A}} & =\sum_{i} n_{i} \frac{\partial \mathcal{H}_{i i}}{\partial x_{A}}+\sum_{i j k l} D_{k l, i j} \frac{\partial\langle i j \mid k l\rangle}{\partial x_{A}} \\
& -\sum_{i j} S_{i j}^{x_{A}} \lambda_{i j}, \quad S_{i j}^{x_{A}}=\sum_{\mu v} C_{\mu i} C_{v j} \frac{\partial \mathcal{S}_{\mu v}}{\partial x_{A}} .
\end{aligned}
$$

The NOs associated to the perturbed geometry are usually expressed as a linear combination of those NOs corresponding to the reference state, so a perturbation of $x_{A}$ up to first order will carry out the next change in the $\phi_{i}$

$$
\phi_{i}+\delta x_{A}\left(\sum_{j} U_{i j}^{x_{A}} \phi_{j}+\sum_{\mu} C_{\mu i} \frac{\partial \zeta_{\mu}}{\partial x_{A}}\right)+\mathcal{O}\left(\delta x_{A}^{2}\right) .
$$

In Eq. (8), $\left\{\zeta_{\mu}\right\}$ are the atomic orbitals, whereas changes in NO coefficients are accounted by standard coupledperturbed coefficients $\left\{U_{i j}^{x_{A}}\right\}$.

The orthonormality relation of the perturbed NOs provides the relationship [6]

$$
\frac{\partial S_{i j}}{\partial x_{A}}=U_{i j}^{x_{A}}+U_{j i}^{x_{A}}+S_{i j}^{x_{A}}=0,
$$

which can be used to derive the relation

$$
\sum_{i j} S_{i j}^{x_{A}} \lambda_{i j}=-2 \sum_{i j} U_{i j}^{x_{A}} \lambda_{i j}
$$


so the electronic energy gradients with respect to Cartesian coordinate $x$ of nucleus $A$ in the NO representation reads as

$$
\begin{aligned}
\frac{d E_{e l}}{d x_{A}} & =\sum_{i} n_{i} \frac{\partial \mathcal{H}_{i i}}{\partial x_{A}}+\sum_{i j k l} D_{k l, i j} \frac{\partial\langle i j \mid k l\rangle}{\partial x_{A}} \\
& +2 \sum_{i j} U_{i j}^{x_{A}} \lambda_{i j} .
\end{aligned}
$$

We may obtain second derivatives of the NOF energy by differentiating Eq. (11) with respect to coordinate $y$ of nucleus $B$, namely,

$$
\begin{aligned}
\frac{d^{2} E_{e l}}{d x_{A} d y_{B}} & =\sum_{i} n_{i} \frac{\partial^{2} \mathcal{H}_{i i}}{\partial x_{A} \partial y_{B}}+\sum_{i j k l} D_{k l, i j} \frac{\partial^{2}\langle i j \mid k l\rangle}{\partial x_{A} \partial y_{B}} \\
& +2 \sum_{i j} U_{i j}^{y_{B}} \lambda_{i j}^{x_{A}}+2 \sum_{i j} \frac{d}{d y_{B}}\left(U_{i j}^{x_{A}} \lambda_{i j}\right) \\
& +\sum_{m} n_{m}^{y_{B}} \frac{\partial}{\partial n_{m}}\left(\frac{d E_{e l}}{d x_{A}}\right) .
\end{aligned}
$$

The first two terms in Eq. (12) contain the explicit derivatives of the core Hamiltonian and the two-electron integrals, respectively. The next two terms arise from the derivatives of $\mathrm{NO}$ coefficients with respect to the nuclear perturbation. Finally, $n_{m}^{y_{B}}$ represents the change in ON $m$ due to perturbation $y_{B}$, so the last term in Eq. (12) accounts for the contribution from the perturbation of the ONs.

Taking into account Eq. (5), the matrix of Lagrange multipliers can be written as

$$
\lambda_{i j}=n_{j} \mathcal{H}_{i j}+2 \sum_{m k l} D_{k l, j m}\langle i m \mid k l\rangle,
$$

so explicit derivatives read as

$$
\lambda_{i j}^{x_{A}}=n_{j} \frac{\partial \mathcal{H}_{i j}}{\partial x_{A}}+2 \sum_{m k l} D_{k l, j m} \frac{\partial\langle i m \mid k l\rangle}{\partial x_{A}} .
$$

Regarding the fourth summation of Eq. (12), a more comprehensive expression can be obtained, namely,

$$
\sum_{i j} \frac{d}{d y_{B}}\left(U_{i j}^{x_{A}} \lambda_{i j}\right)=\sum_{i j}\left\{\frac{d U_{i j}^{x_{A}}}{d y_{B}} \lambda_{i j}+U_{i j}^{x_{A}} \frac{d \lambda_{i j}}{d y_{B}}\right\},
$$

where the first term in Eq. (15) is given by [6]

$$
\frac{d U_{i j}^{x_{A}}}{d y_{B}}=U_{i j}^{x_{A} y_{B}}-\sum_{k} U_{i k}^{y_{B}} U_{k j}^{x_{A}} .
$$

By using Eq. (9) together with the orthonormality rela- tion of the NOs we arrive at [6]

$$
\begin{gathered}
\frac{\partial^{2} S_{i j}}{\partial x_{A} \partial y_{B}}=U_{i j}^{x_{A} y_{B}}+U_{j i}^{x_{A} y_{B}}-\sum_{m}\left\{S_{i m}^{y_{B}} S_{j m}^{x_{A}}+S_{j m}^{y_{B}} S_{i m}^{x_{A}}\right. \\
\left.-U_{i m}^{y_{B}} U_{j m}^{x_{A}}-U_{j m}^{y_{B}} U_{i m}^{x_{A}}\right\}+\sum_{\mu v} C_{\mu i} C_{v j} \frac{\partial^{2} \mathcal{S}_{\mu v}}{\partial x_{A} \partial y_{B}}=0
\end{gathered}
$$

then

$$
\begin{gathered}
2 \sum_{i j} U_{i j}^{x_{A} y_{B}} \lambda_{i j}=\sum_{i j} \lambda_{i j}\left(\sum _ { m } \left\{S_{i m}^{y_{B}} S_{j m}^{x_{A}}+S_{j m}^{y_{B}} S_{i m}^{x_{A}}\right.\right. \\
\left.\left.-U_{i m}^{y_{B}} U_{j m}^{x_{A}}-U_{j m}^{y_{B}} U_{i m}^{x_{A}}\right\}-\sum_{\mu v} C_{\mu i} C_{v j} \frac{\partial^{2} \mathcal{S}_{\mu v}}{\partial x_{A} \partial y_{B}}\right) .
\end{gathered}
$$

The derivative of Lagrange multipliers is obtained differentiating Eq. (13)

$$
\frac{d \lambda_{i j}}{d y_{B}}=\lambda_{i j}^{y_{B}}+\sum_{k} U_{k i}^{y_{B}} \lambda_{k j}+\sum_{k l} U_{k l}^{y_{B}} Y_{i j k l},
$$

where

$$
\begin{aligned}
Y_{i j k l} & =n_{j} \delta_{j l} \mathcal{H}_{i k}+2 \sum_{m n} D_{l n, j m}\langle i m \mid k n\rangle \\
& +4 \sum_{m n} D_{m n, j l}\langle i k \mid m n\rangle .
\end{aligned}
$$

In Eq. (19), the response from ONs has been omitted since it is included later. Overall the fourth summation in Eq. (12) is given by

$$
\begin{aligned}
& \sum_{i j} \frac{d}{d y_{B}}\left(U_{i j}^{x_{A}} \lambda_{i j}\right)=\sum_{i j}\left\{U_{i j}^{x_{A} y_{B}} \lambda_{i j}+U_{i j}^{x_{A}} \lambda_{i j}^{y_{B}}\right. \\
&\left.+\sum_{k l} U_{i j}^{x_{A}} U_{k l}^{y_{B}} Y_{i j k l}\right\} .
\end{aligned}
$$

In the last summation of Eq. (12), the derivatives with respect to the occupancies read as

$$
\begin{aligned}
\frac{\partial}{\partial n_{m}}\left(\frac{\partial E_{e l}}{\partial x_{A}}\right) & =\frac{\partial \mathcal{H}_{m m}}{\partial x_{A}}+2 \sum_{i j} U_{i j}^{x_{A}} \frac{\partial \lambda_{i j}}{\partial n_{m}} \\
& +\sum_{i j k l} \frac{\partial D_{k l, i j}}{\partial n_{m}} \frac{\partial\langle i j \mid k l\rangle}{\partial x_{A}},
\end{aligned}
$$

where

$$
\frac{\partial \lambda_{i j}}{\partial n_{m}}=\delta_{m j} \mathcal{H}_{i j}+2 \sum_{r k l} \frac{\partial D_{k l, j r}}{\partial n_{m}}\langle i r \mid k l\rangle .
$$

Note that $\partial D_{k l, j r} / \partial n_{m}$ is determined by the given twoparticle RDM reconstruction $D\left[n_{i}, n_{j}, n_{k}, n_{l}\right]$ (see Eq. 21). Substituting Eqs. (20) and (21) into Eq. (12), we 
obtain the general expression for the Hessian in the NO representation, namely,

$$
\begin{array}{r}
\frac{d^{2} E_{e l}}{d x_{A} d y_{B}}=\sum_{i} n_{i} \frac{\partial^{2} \mathcal{H}_{i i}}{\partial x_{A} \partial y_{B}}+\sum_{i j k l} D_{k l, i j} \frac{\partial^{2}\langle i j \mid k l\rangle}{\partial x_{A} \partial y_{B}} \\
+2 \sum_{i j}\left(U_{i j}^{y_{B}} \lambda_{i j}^{x_{A}}+U_{i j}^{x_{A}} \lambda_{i j}^{y_{B}}+U_{i j}^{x_{A} y_{B}} \lambda_{i j}\right) \\
+2 \sum_{i j k l} U_{i j}^{x_{A}} U_{k l}^{y_{B}} Y_{i j k l}+\sum_{m} n_{m}^{y_{B}}\left(\frac{\partial \mathcal{H}_{m m}}{\partial x_{A}}\right. \\
\left.+2 \sum_{i j} U_{i j}^{x_{A}} \frac{\partial \lambda_{i j}}{\partial n_{m}}+\sum_{i j k l} \frac{\partial D_{k l, i j}}{\partial n_{m}} \frac{\partial\langle i j \mid k l\rangle}{\partial x_{A}}\right) .
\end{array}
$$

In contrast to first-order energy derivatives, the calculation of the analytic Hessian requires the knowledge of NOs and ONs at the perturbed geometry, expressed in Eq. (22) by coefficients $U$ and $n_{m}^{y_{B}}$, respectively. Both magnitudes are obtained from the solution of coupled perturbed equations which are the result of deriving the variational conditions (445). It is worth noting that in the case of Eq. (5), it is more convenient to use its combination with its Hermitian conjugate equation that gives us the variational condition on the Hermiticity of Lagrange multipliers $\left(\lambda-\lambda^{\dagger}=0\right)$.

\section{COUPLED-PERTURBED EQUATIONS}

Coupled perturbed equations for NOs and ONs were derived by Pernal and Baerends 17 to obtain the linear response of $\Gamma$ in a problem with a one-electron static perturbation in the Hamiltonian. In particular, these equations were employed in the calculation of the static polarizabilities of atoms and molecules. The formalism was later extended by Giesbertz [18] to deal with pinned ONs.

Here we present the coupled perturbed equations for NOs and ONs considering from the beginning that NOs have an explicit dependence on the perturbation (Eq. 8) through the position dependence of the basis functions. Therefore, instead of considering an anti-Hermitian $U$ matrix as done in Refs. [17, 18], standard coupledperturbed coefficients are related with the overlap matrix $S$ by Eq. (9). In addition, the existence of a generalized Fock matrix has not been assumed in the present derivation. Our coupled-perturbed equations are obtained from the Euler equations (4.5), which are valid for any approximate NOF.

For real orbitals, at the extremum, the total derivatives of the variational condition on the Hermiticity of Lagrange multipliers vanishes,

$$
\frac{d}{d x_{A}}\left(\lambda_{i j}-\lambda_{j i}\right)=0 .
$$

Taking into account Eqs. (19) and (22), Eq. (24) can be rewritten as

$$
\begin{gathered}
\lambda_{i j}^{x_{A}}-\lambda_{j i}^{x_{A}}+\sum_{k}\left(U_{k i}^{x_{A}} \lambda_{k j}-U_{k j}^{x_{A}} \lambda_{k i}\right)+\sum_{k l}\left(U_{k l}^{x_{A}}\right. \\
\left.Y_{i j k l}-U_{k l}^{x_{A}} Y_{j i k l}\right)+\sum_{k}\left(\frac{\partial \lambda_{i j}}{\partial n_{k}}-\frac{\partial \lambda_{j i}}{\partial n_{k}}\right) n_{k}^{x_{A}}=0 .
\end{gathered}
$$

Eq. (9) can be used to simplify first and second summations in Eq. (25), namely,

$$
\begin{aligned}
\sum_{k} U_{k i}^{x_{A}} \lambda_{k j} & =\sum_{k>l}\left[U_{k l}^{x_{A}}\left(\lambda_{k j} \delta_{l i}-\lambda_{l j} \delta_{k i}\right)\right. \\
& \left.-S_{k l}^{x_{A}} \lambda_{l j} \delta_{k i}\right]-\frac{1}{2} \sum_{k} S_{k k}^{x_{A}} \lambda_{k j} \delta_{k i}, \\
\sum_{k l} U_{k l}^{x_{A}} Y_{i j k l} & =\sum_{k>l}\left[U_{k l}^{x_{A}}\left(Y_{i j k l}-Y_{i j l k}\right)\right. \\
& \left.-S_{k l}^{x_{A}} Y_{i j l k}\right]-\frac{1}{2} \sum_{k} S_{k k}^{x_{A}} Y_{i j k k} .
\end{aligned}
$$

Accordingly, Eq. 25) can be rewritten as

$$
\begin{gathered}
\lambda_{i j}^{x_{A}}-\lambda_{j i}^{x_{A}}+\sum_{k}\left(\frac{\partial \lambda_{i j}}{\partial n_{k}}-\frac{\partial \lambda_{j i}}{\partial n_{k}}\right) n_{k}^{x_{A}} \\
-\frac{1}{2} \sum_{k} S_{k k}^{x_{A}}\left(\delta_{k i} \lambda_{k j}-\delta_{k j} \lambda_{k i}+Y_{i j k k}-Y_{j i k k}\right) \\
+\sum_{k>l} U_{k l}^{x_{A}}\left(\delta_{l i} \lambda_{k j}-\delta_{k i} \lambda_{l j}-\delta_{l j} \lambda_{k i}+\delta_{k j} \lambda_{l i}\right. \\
\left.\quad+Y_{i j k l}-Y_{i j l k}-Y_{j i k l}+Y_{j i l k}\right) \\
-\sum_{k>l} S_{k l}^{x_{A}}\left(\delta_{k i} \lambda_{l j}-\delta_{k j} \lambda_{l i}+Y_{i j l k}-Y_{j i l k}\right)=0
\end{gathered}
$$

Let us now consider the Eq. (44) involving derivatives with respect to ONs. A perturbation up to first order transforms it into

$$
\begin{aligned}
& \frac{\partial \mathcal{H}_{m m}}{\partial x_{A}}+\sum_{i j k l} \frac{\partial D_{k l, i j}}{\partial n_{m}} \frac{\partial\langle i j \mid k l\rangle}{\partial x_{A}}+\sum_{r i j k l} \frac{\partial^{2} D_{k l, i j}}{\partial n_{m} \partial n_{r}}\langle i j \mid k l\rangle n_{r}^{x_{A}} \\
& +2 \sum_{i j}\left[U_{i j}^{x_{A}}\left(\delta_{j m} \mathcal{H}_{i j}+2 \sum_{r k l} \frac{\partial D_{k l, j r}}{\partial n_{m}}\langle i r \mid k l\rangle\right)\right]=\mu^{x_{A}} .
\end{aligned}
$$

Taking into account Eq. (9), Eq. (29) can be rewritten in compact form as

$$
\sum_{r} W_{m r} n_{r}^{x_{A}}+\sum_{i>j} U_{i j}^{x_{A}}\left(E_{i j}^{m}-E_{j i}^{m}\right)=F_{m}^{x_{A}}
$$


where

$$
\begin{aligned}
F_{m}^{x_{A}} & =\mu^{x_{A}}-\left(\frac{\partial \mathcal{H}_{m m}}{\partial x_{A}}+\sum_{i j k l} \frac{\partial D_{k l, i j}}{\partial n_{m}} \frac{\partial\langle i j \mid k l\rangle}{\partial x_{A}}\right) \\
& +\sum_{i>j} S_{i j}^{x_{A}} E_{j i}^{m}+\frac{1}{2} \sum_{i} S_{i i}^{x_{A}} E_{i i}^{m}, \\
E_{i j}^{m}= & 2 \delta_{j m} \mathcal{H}_{i j}+4 \sum_{r k l} \frac{\partial D_{k l, j r}}{\partial n_{m}}\langle i r \mid k l\rangle, \\
W_{m r} & =\sum_{i j k l} \frac{\partial^{2} D_{k l, i j}}{\partial n_{m} \partial n_{r}}\langle i j \mid k l\rangle .
\end{aligned}
$$

Note that $E_{i j}^{m}$ relates to $\partial \lambda_{i j} / \partial n_{m}$ by a factor $1 / 2$ according to Eq. (22), so Eqs. (28) and (30) can bring together to obtain the complete expression for the coupledperturbed NOF equations

$$
\begin{gathered}
\forall_{i>j} \sum_{k>l} A_{i j, k l} U_{k l}^{x_{A}}+\left(E_{i j}^{k}-E_{j i}^{k}\right) n_{k}^{x_{A}}=B_{i j}^{x_{A}} \\
\forall_{i} \quad \sum_{k>l}\left(E_{k l}^{i}-E_{l k}^{i}\right) U_{k l}^{x_{A}}+W_{i k} n_{k}^{x_{A}}=F_{i}^{x_{A}}
\end{gathered}
$$

where

$$
\begin{aligned}
A_{i j, k l} & =\delta_{l i} \lambda_{k j}-\delta_{k i} \lambda_{l j}-\delta_{l j} \lambda_{k i}+\delta_{k j} \lambda_{l i} \\
& +Y_{i j k l}-Y_{i j l k}-Y_{j i k l}+Y_{j i l k}, \\
B_{i j}^{x_{A}}= & \sum_{k>l} S_{k l}^{x_{A}}\left(\delta_{k i} \lambda_{l j}-\delta_{k j} \lambda_{l i}+Y_{i j k l}-Y_{j i l k}\right) \\
+ & \frac{1}{2} \sum_{k} S_{k k}^{x_{A}}\left(\delta_{k i} \lambda_{k j}-\delta_{k j} \lambda_{k i}+Y_{i j k k}-Y_{j i k k}\right) \\
- & \lambda_{i j}^{x_{A}}+\lambda_{j i}^{x_{A}} .
\end{aligned}
$$

It is worth noting that the coupled-perturbed equations given by Eq. (31) are totally general and can be easily implemented, so that an expression for the reconstructed $D\left[n_{i}, n_{j}, n_{k}, n_{l}\right]$ is only required. The here presented formulation of such equations exploits Eq. (9) to calculate only necessary $U$ coefficients, namely, the lower (or upper) block of matrix $U$.

The matricial form of Eq. (31) is

$$
\left(\begin{array}{cc}
A & E-E^{\dagger} \\
E-E^{\dagger} & W
\end{array}\right)\left(\begin{array}{c}
U^{x_{A}} \\
n^{x_{A}}
\end{array}\right)=\left(\begin{array}{c}
B^{x_{A}} \\
F^{x_{A}}
\end{array}\right),
$$

where $E^{\dagger}$ represents conjugate transpose operation only acting on the subindexes, and it makes clear the symmetric nature of the square matrix. The latter has to be computed and inverted only once, since it is independent of the perturbation $\delta x_{A}$, and presents only dependence on non-perturbed NOs and ONs.

\section{CLOSING REMARKS}

Simple analytic expressions have been derived for computation of the second-order energy derivatives with respect to nuclear displacements in the context of the natural orbital functional theory. An alternative expression for analytic gradients in terms of the NOs is given as well. In contrast to first-order energy derivatives, the calculation of the analytic Hessian requires the knowledge at the perturbed geometry of NOs and ONs, which are obtained from the solution of coupled-perturbed equations.

The coupled-perturbed equations were obtained from the corresponding variational Euler equations considering that also basis functions have explicit dependence on the geometry perturbations. Consequently, the linear response of both NOs and ONs to non-external perturbations of the Hamiltonian, as in the case of nuclear geometry displacements, can be easily obtained by solving a set of equations that only need to specify the reconstruction of the second-order RDM in terms of the ONs.

In geometry optimization problems, the algorithms that employ the Hessian knowledge are superior with respect to methods that use only the gradient. The Hessian can be used for the most efficient search of an extremum, and to test whether an extremum is a minimum or maximum too. The formulas here presented constitute the groundwork for practical calculations related to second-order energy derivatives with respect to nuclear displacements, such as computation of harmonic vibrational frequencies and thermochemical analysis.

\section{Acknowledgments}

Financial support comes from Eusko Jaurlaritza (Ref. IT588-13) and Ministerio de Economia y Competitividad (Ref. CTQ2015-67608-P). One of us (I.M.) is grateful to Vice-Rectory for research of the UPV/EHU for the PhD. grant (PIF//15/043). The SGI/IZO-SGIker UPV/EHU is gratefully acknowledged for generous allocation of computational resources.
[1] I. Papai, A. St-Amant, J. Ushio, and D. Salahub, Int. J. Quantum Chem. 38, 29 (1990).
[2] M. Frisch, M. Head-Gordon, and J. Pople, Chem. Phys. Lett. 141, 189 (1990). 
[3] J. Russel Thomas, J. DeLeeuw Bradley, George Vacek, T. Daniel Crawford, J. Chem. Phys. 99, 403 (1993).

[4] M. W. Wong, Chem. Phys. Lett. 256, 391 (1996).

[5] P. Pulay, WIREs Comput. Mol. Sci. 4, 169 (2014).

[6] Y. Yamaguchi and H. F. Schaefer, Analytic Derivative Methods in Molecular Electronic Structure Theory : A New Dimension to Quantum Chemistry and its Applications to Spectroscopy (John Wiley and Sons, LTD, 2011).

[7] I. Mitxelena and M. Piris, J. Chem. Phys. 144, 204108 (2016).

[8] D. A. Mazziotti, in Reduced-Density-Matrix Mechanics: with applications to many-electron atoms and molecules, edited by D. A. Mazziotti (John Wiley and Sons, Hoboken, New Jersey, USA, 2007), chap. 3, pp. 21-59, 1st ed.

[9] A. Y. Sokolov, J. J. Wilke, A. C. Simmonett, and H. F. Schaefer, J. Chem. Phys. 137 (2012).

[10] M. Piris and J. M. Ugalde, Int. J. Quantum Chem. 114, 1169 (2014), (and references therein).

[11] A. J. Coleman, Rev. Mod. Phys. 35, 668 (1963).

[12] M. Piris, in Reduced-Density-Matrix Mechanics: with applications to many-electron atoms and molecules, edited by D. A. Mazziotti (John Wiley and Sons, Hoboken, New Jersey, USA, 2007), chap. 14, pp. 387-427.
[13] M. Piris, in Many-body approaches at different scales: a tribute to N. H. March on the ocasion of his 90th birthday, edited by G. G. N. Angilella and C. Amovilli (Springer, New York, USA, 2017), chap. 22, pp. 231-247.

[14] K. Pernal and K. J. H. Giesbertz, Top Curr Chem. 368, 125 (2016), (and references therein).

[15] I. Mitxelena and M. Piris, J. Chem. Phys. 146, 014102 (2017).

[16] M. Piris and J. M. Ugalde, J. Comput. Chem. 30, 2078 (2009).

[17] K. Pernal and E. J. Baerends, J. Chem. Phys. 124, 014102 (2006).

[18] K. J. H. Giesbertz, Ph.D. thesis, Vrije Universiteit, Amsterdam, The Netherlands (2010).

[19] David A. Mazziotti, Phys. Rev. Lett. 117, 153001 (2016).

[20] Anthony W. Schlimgen, Charles W. Heaps, David A. Mazziotti, J. Phys. Chem. Lett., 7 (4), 627-631 (2016).

[21] Alexandra R. McIsaac, David A. Mazziotti, Phys. Chem. Chem. Phys., 19, 4656-4660 (2017).

[22] Andrew J. S. Valentine, David A. Mazziotti, Chem. Phys. Lett., 685, 300-304 (2017). 